\title{
Development of superconducting transition edge sensors based on electron-phonon decoupling
}

\author{
Nikhil Jethava*ab ${ }^{\text {ab }}$ James Chervenak ${ }^{\mathrm{a}}$, Ari-David Brown ${ }^{\mathrm{ac}}$, Dominic Benford ${ }^{\mathrm{a}}$, Gunther Kletetschka ${ }^{\mathrm{a}}$, \\ Vilem Mikula $a^{\text {ad }}$, Kongpop U-yen ${ }^{\mathrm{a}}$ \\ ${ }^{a}$ NASA Goddard Space Flight Ctr, Greenbelt, MD, USA 20771; \\ ${ }^{\mathrm{b}}$ Global Science and Technology, 7855 Walker Drive, Greenbelt, MD 20770; \\ ${ }^{c}$ MEI Technologies, Inc., 7404 Executive Pl \#400, Lanham, MD 20706; \\ ${ }^{\mathrm{d}}$ Department of Physics, Catholic University of America, 200 Hannan Hall, Washington, DC 20064
}

\begin{abstract}
We have successfully fabricated a superconducting transition edge sensor (TES), bolometer that centers on the use of electron-phonon decoupling (EPD) for thermal isolation. We have selected a design approach that separates the two functions of far-infrared and $\mathrm{THz}$ radiative power absorption and temperature measurement, allowing separate optimization of the performance of each element. We have integrated molybdenum/gold $(\mathrm{Mo} / \mathrm{Au})$ bilayer TES and ion assisted thermally evaporated (IAE) bismuth (Bi) films as radiation absorber coupled to a low-loss microstripline from niobium $(\mathrm{Nb})$ ground plane to a twin-slot antenna structure. The thermal conductance $(\mathrm{G})$ and the time constant for the different geometry device have been measured. For one such device, the measured $G$ is $1.16 \times 10^{-10} \mathrm{~W} / \mathrm{K}\left( \pm 0.61 \times 10^{-}\right.$ ${ }^{10} \mathrm{~W} / \mathrm{K}$ ) at $60 \mathrm{mK}$, which corresponds to noise equivalent power (NEP) $=1.65 \times 10^{-18} \mathrm{~W} / \sqrt{ } \mathrm{Hz}$ and time constant of $\sim 5 \mu \mathrm{s}$.
\end{abstract}

Keywords: Bolometer, Thermal Conductance, Electron-Phonon decoupling, Transition Edge Sensors

\section{INTRODUCTION}

A TES bolometer has a higher sensitivity-response time product than a semiconducting bolometer with the same geometry due to the strong negative electrothermal feedback intrinsic in typical a voltage-biased TES ${ }^{1,2,3}$. TES bolometers are inherently low impedance devices, so they are well matched to being read out by DC SQUID amplifiers. SQUID amplifiers are mature and have been used extensively for TES bolometer arrays in formats of up to $\sim 1000$ pixels. Because SQUIDs operate at the base temperature of the bolometer, they can be coupled very closely, removing the complex interfaces necessary with semiconducting bolometers and FET amplifiers.

Most TES employ fragile mechanical isolation in order to decouple the detector temperature from a cold stage temperature in order to achieve high sensitivity. Since, the electronic and phononic temperatures of the sensor can be significantly different, one can eliminate the need for mechanical isolation if $\mathrm{T}<<1 \mathrm{~K}$ and sensors volume is small $(\sim 10 \mathrm{~s}$ $\mu \mathrm{m}^{2}$ ). The weak interaction (thermal coupling) between the electrons and the phonons in metals allows a large temperature rise of the electrons for small input power. The main advantage of using this effect for thermal isolation rather than fragile mechanical isolation is that the bolometer does not require micromachining or assembly. Instead, the radiative power is coupled directly into the electrons in the metal, and the temperature of these electrons is measured directly.

Detectors that achieve the required NEP will be designed taking into account this effect, called electron-phonon decoupling (EPD). This method can produce low NEPs at achievable temperatures while enabling large-format arrays with reasonable fill factor and rapid thermal response time. 


\section{FABRICATION}
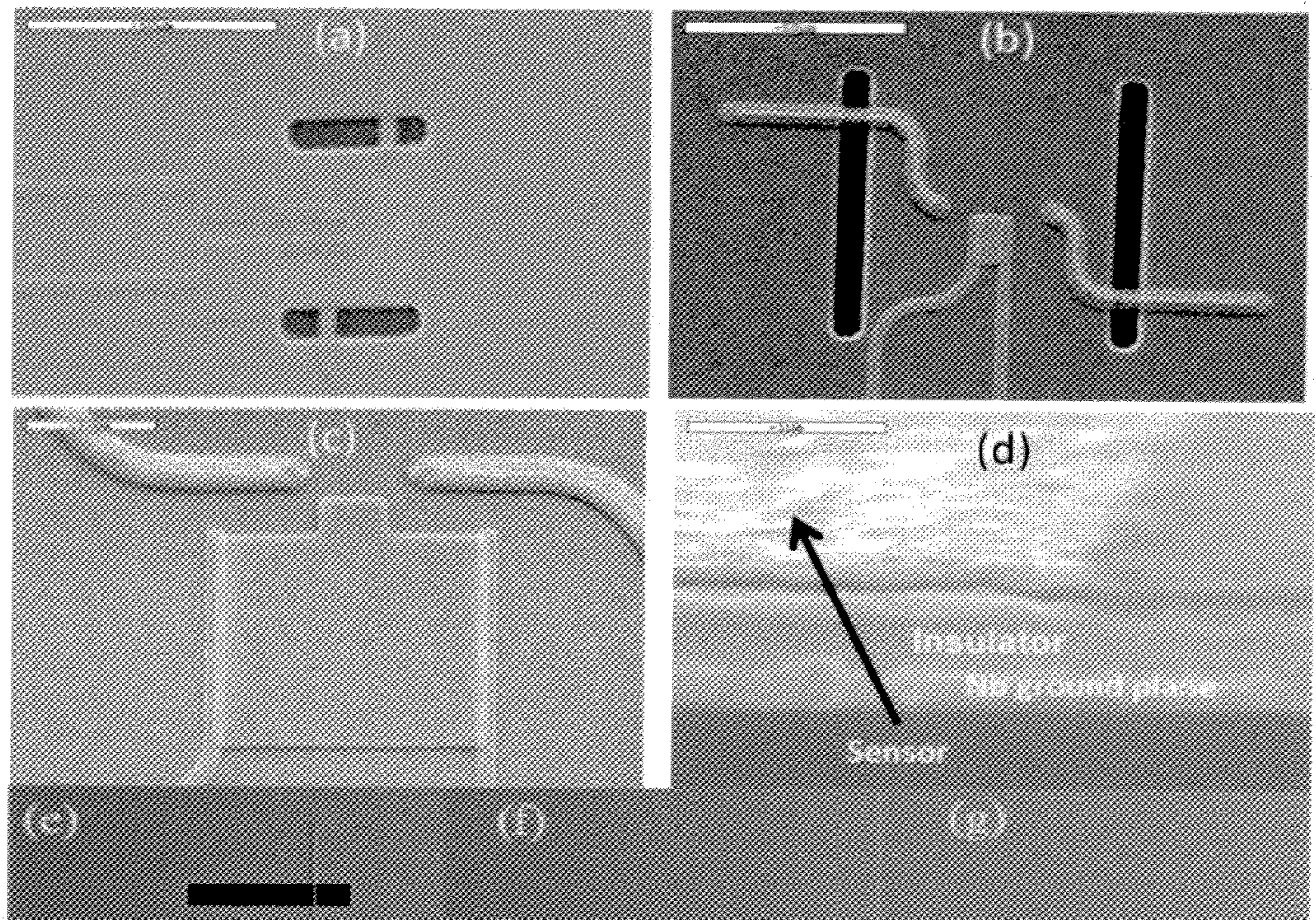

.
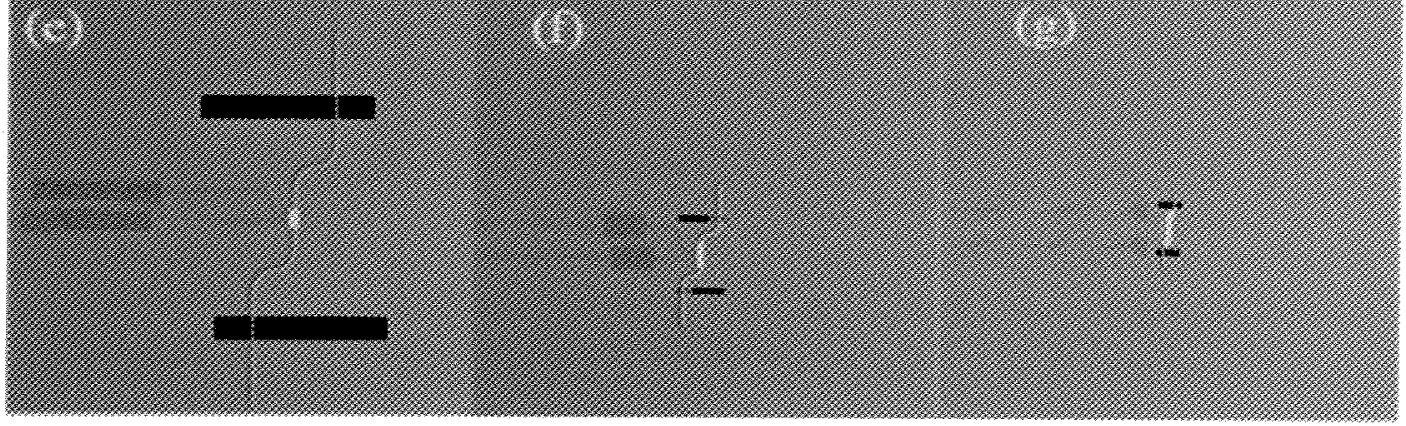

Figure 1. (a) SEM image of $25 \mu \mathrm{m}^{2}$ device. (b) SEM image of $50 \mu \mathrm{m}^{2}$ device. (c) SEM image of $1225 \mu \mathrm{m}^{2}$ device. (d) Image shows the Nb ground plane, an insulator and Mo/Au TES sensor. (e), (f) and (g) are images of $300 \mathrm{GHz}$, $1 \mathrm{THz}$, and $3 \mathrm{THz}$ devices, respectively. For (e), (f) and $(\mathrm{g})$, a thin layer of $\mathrm{Bi}$ is deposited to act as an radiation absorber.

\subsection{Electron-Phonon decoupling}

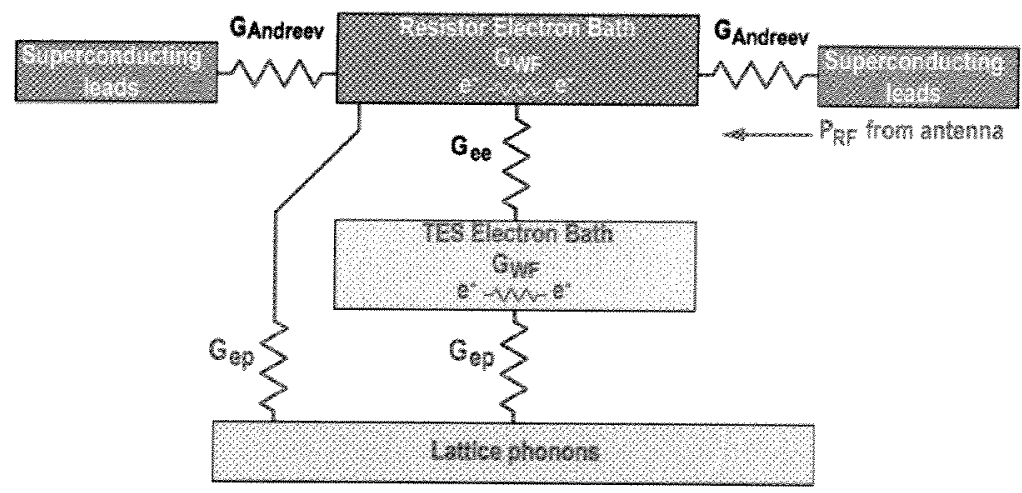

Figure 2. The thermal block diagram of the proposed EPD. 


\subsection{Detector design}

We have integrated a small volume resistor, a similarly small volume TES, and a low-loss microstripline to an antenna structure to fabricate EPD TES receiver. We have chosen to implement a twin-slot antenna because of its symmetric beam pattern, high efficiency at short wavelengths, good rejection of out-of-band radiation, and the convenient ground plane that can be used to shield DC wiring. Short sections of microstrip with low loss at THz frequencies (high quality $\mathrm{Au}$, which modeling shows will have $\sim 0.1 \mathrm{~dB}$ loss) will allow manipulation of the placement of circuit components for optical coupling purposes. Nb bias line chokes allow on-chip filtering of the TES bias lines and improved shielding. Figure 2 shows the schematic layout of complete EPD detector.

Niobium $(\mathrm{Nb})$ will be employed as both the ground plane and stripline material in the lower frequency range of interest $(300-500 \mathrm{GHz})$ because of its superconductivity. However, at frequency above its energy gap $\mathrm{Nb}$ acts like a normal metal with relatively high impedance. Consequently, gold, which is a low impedance normal metal film will be used, and retains low radiation loss properties over a wide frequency range. In order to minimize both radiation loss and high reactance, we aimed to maximize the gold microstrip sheet conductance so as to permit a variety of microstrip geometries. We have recently these devices and plans for testing are underway ${ }^{10}$.

We use ion assisted thermally evaporated (IAE) Bismuth (Bi) as radiation absorber ${ }^{10}$. It is found that the residual resistance ratio of the IAE $\mathrm{Bi}$ is an order of magnitude higher than that of evaporated (TE) $\mathrm{Bi}$. IAE Bi can be useful for antenna-coupled sub-millimeter bolometers, because it is resistive (i.e., it has a low reactance) to high frequencies. This contrasts with $\mathrm{TE} \mathrm{Bi}$, which is reactive at frequencies as low as $1 \mathrm{THz}$. The improved performance of IAE Bi over that of TE Bi might be attributed to its smaller grain structure and/or lower impurity concentration. Because the IAE Bi resistivity $r$ is a thickness-independent quantity, we can easily estimate the noise power $P$ of a termination resistor fabricated with this material ${ }^{10}$.

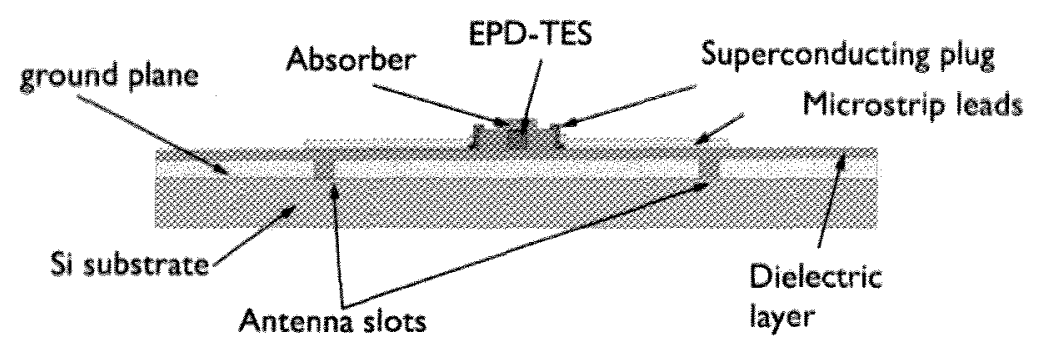

Figure 3. This schematic side view of the circuit fabrication concept for producing the EPD TES devices.

\subsection{Detector fabrication}

Electron-phonon decoupled TES were fabricated on 4" $\mathrm{Si}(001)$ wafers. One notable aspect of our sensor design is the absence of membranes, which greatly simplifies the fabrication process. An Nb ground plane was DC magnetron sputter deposited and etched, via a $\mathrm{CF}_{4} / \mathrm{O}_{2}$ plasma, in regions designated for the slot antennas. A silicon dioxide dielectric was next applied via electron cyclotron resonance plasma enhanced chemical vapor deposition. This was a low temperature process, in which the wafer temperature never exceeded $180 \mathrm{C}$, and enabled a conformal dielectric coating. Niobium microstrip lines were then deposited and etched in a manner similar to that used to fabricate the ground plane. The TES consisted of a Mo/Au bilayer film and was deposited on top of the silicon dioxide using electron beam deposition and $\mathrm{DC}$ magnetron sputtering for the $\mathrm{Mo}$ and $\mathrm{Au}$, respectively. The $\mathrm{Mo} / \mathrm{Au}$ deposition was conducted inside a custom set of vacuum chambers which are connected via a common loadlock. This configuration allowed for a high degree of $T_{c}$ uniformity and reproducibility of the TES bilayer film across the wafer, because the Mo surface was never exposed to atmosphere. The TES were delineated using a positive photoresist mask, and ion milling and reactive ion etching, with a $\mathrm{CF}_{4} / \mathrm{O}_{2}$ plasma, were used to etch the $\mathrm{Au}$ and Mo, respectively. Niobium bias leads were then deposited and etched in a manner similar to that used to fabricate the ground plane and were aligned so as to overlap the TES by $0.5 \mu \mathrm{m}$ and 1.0 $\mu \mathrm{m}$ for $25,50 \mu \mathrm{m}$ and $100,300,1225 \mu \mathrm{m}$ devices, respectively (see Figure 1). Aluminum pads were deposited through a liftoff mask and made contact to the bias leads. 
The final step in the fabrication process involved deposition of a bismuth termination resistor. Bismuth is a highly reactive material, which makes it susceptible to subsequent fabrication processes, e.g., photoresist application and developing. Consequently, we first patterned a PMGI lift-off mask prior to IAE deposition of $99.999 \%$ bismuth. The bismuth was then lifted-off in acetone. Fabrication of the TES diagnostic devices, whose purpose was to solely characterize the TES, was a much more simple process. This process only consisted of TES deposition on $\mathrm{SiO}_{2}$-coated $\mathrm{Si}(0 \mathrm{O} 1)$ wafer and deposition of the $\mathrm{Nb}$ bias leads and $\mathrm{Al}$ pads.

\section{EXPERIMENTAL RESULTS}

We have fabricated devices in different geometries. Table 1 presents details of these devices and their characteristic parameters. We used Resistance-Temperature curves: $G(T)=I^{2} R_{n} /(\nabla T)$ (Figure 4) to determine the thermal conductance for each device ${ }^{6,8,9}$. Here, $G(T)$ is the thermal conductance at the temperature $T, R_{n}$ is the normal state resistance of sensor, and $\nabla T$ is the hysteresis in the Resistance-Temperature curve while increasing and decreasing the fridge temperature.

Table 1. The surface area, superconducting transition temperature (Tc) and normal state resistance (Rn) for four different detector geometries are listed. The TES was fabricated using bilayer of molybdenum (Mo) and gold (Au) material. The Mo thickness $=50 \mathrm{~nm}$ and the Au thickness $=20 \mathrm{~nm}$ for all the devices. Tc and Rn values are calculated at lowest excitation current, i.e, $0.5 \mu \mathrm{A}$.

\begin{tabular}{|c|c|c|c|c|}
\hline Device & $\begin{array}{c}\text { Surface area } \\
\left(\boldsymbol{\mu m}^{2}\right)\end{array}$ & $\begin{array}{c}\text { On Nb Ground } \\
\text { plane }\end{array}$ & $\begin{array}{c}\mathbf{R n} \\
(\boldsymbol{\Omega} / \square)\end{array}$ & $\begin{array}{c}\text { Tc } \\
(\mathbf{m} \mathbf{K})\end{array}$ \\
\hline A & 25 & No & 0.28 & 410 \\
\hline B & 50 & No & 0.45 & 559 \\
\hline C & 300 & Yes & 0.36 & 232 \\
\hline D & 1225 & Yes & 0.36 & 341 \\
\hline
\end{tabular}

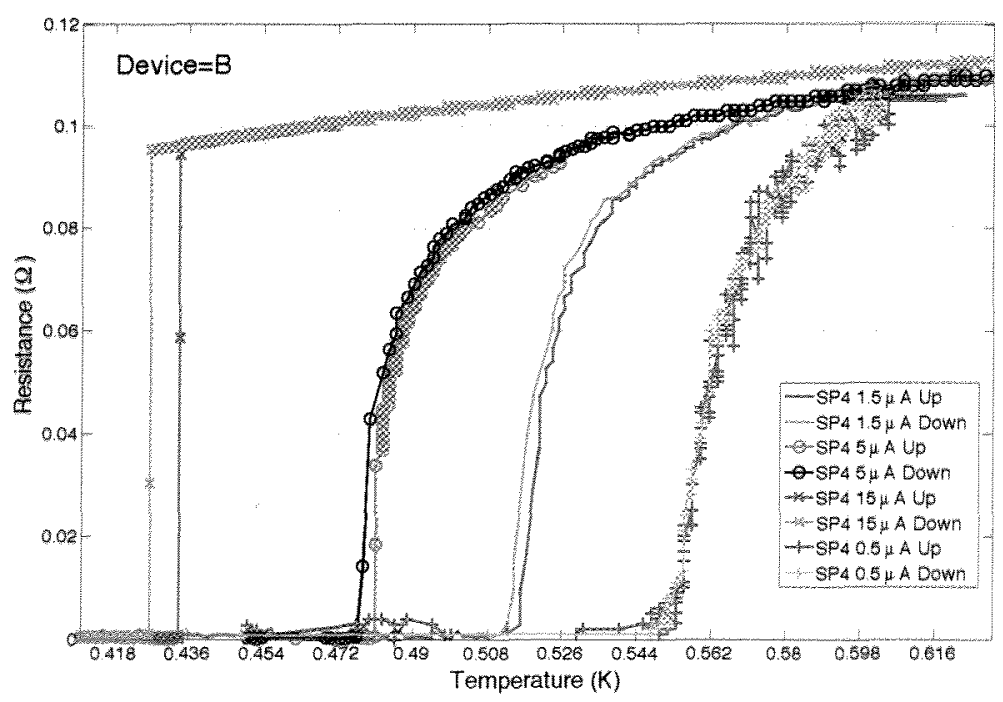

Figure 4. Superconducting transition curves of Device B at different bias current. The "Up" and "Down" in the plot corresponds to increasing and decreasing the bath temperature of cryostat, respectively. We used Adiabatic Demagnetization Refrigerator (ADR) with dry pulse tube cryostat to reach to the base temperature of $50 \mathrm{mK}$. Thin $\mathrm{Nb}$ foils and Lead tape magnetically shield the device package.

The proposed EPD approach will employ a thermal diagram (Figure 1) and electrical connection (Figure 2). An antenna couples power into an absorbing resistor, which thermalizes the power into the electron bath in a time given by its internal thermal conductance, $G_{W F}$. This power is communicated through $G_{\text {electron-electron }}\left(G_{e e}\right)$ to the TES. The power then 
dissipates into the lattice phonons through a weak thermal link $G_{\text {electron-phonon }}\left(G_{e p}\right)$. Provided that $G_{e p} \ll G_{W F}$ and $G_{e p} \ll G_{e e}$, which we calculate to be true for temperatures below $\sim 400 \mathrm{mK}$, the sensitivity and time constant of the detector are then dominated by $G_{e p}$. This approach is very similar to that used by other research groups ${ }^{8,9}$, who have tested EPD devices fabricated by our group, yielding accurate knowledge of materials parameters related to our processes.

The power conducted between electrons at a temperature $T_{e}$ and phonons at a temperature of $T_{p}$ is given by:

$$
P_{e p}=\Sigma V\left(T_{e}^{5}-T_{p}^{5}\right)
$$

where $V$ is the volume of the metal and $\mathrm{S}$ is a material-dependent constant. A typical value of $\Sigma$ for gold ${ }^{9}$ at modest cryogenic temperatures $(<1 \mathrm{~K})$ is thought to be around $5 \times 10^{8} \mathrm{~W} / \mathrm{m}^{3} / \mathrm{K}^{8}$.

Differentiating Eq. (1) provides the thermal conductance:

$$
G_{e p}=5 \Sigma V T^{4}
$$

As the film thickness is much smaller than the thermal acoustic wavelength as is certainly the case in these experiments, the Kapitza conductance is underestimated by acoustic mismatch arguments. [here you seem to be saying that acoustic mismatch arguments don't accurately model Kapitza conductance; if not, what does? You should clarify this] As has been determined experiment by many other groups ${ }^{6,7,8,9}$, the principal impedance for heat flow is the electron phonon coupling. The electron phonon conductance depends on $T^{4}$. Figure 5 plots the measured $\mathrm{G}$ values for all the devices at different base temperature, and $T^{4}$ fit to the data.

As seen from the Figure 5, Device D does not fit very well to $T^{4}$ fit. The higher order $T$ term $\left(\mathrm{T}^{5}\right)$ could fit Device $\mathrm{D}$ data more precisely. In the cases in which electron scattering mechanisms are governed by impurities, defects, or boundaries of the film, the shortened mean free path of the electrons should affect the electron-phonon coupling in the disordered metals. In a disordered film, the electron mean free path $l_{e}$ is very short compared to the wave length of phonons; $q . l_{e} \ll 1$ with $q$ as the phonon wave vector. In this dirty limit, M. Yu. Reizer and A. V. Sergeev ${ }^{11}$ suggested that the thermal conductivity should be proportional to $\mathrm{T}^{5}$, not $\mathrm{T}^{4}$. Since, Device $\mathrm{D}$ has larger sensors area, the distortions in the film can produce imperfections in the film, which can lead to higher $G$ values.

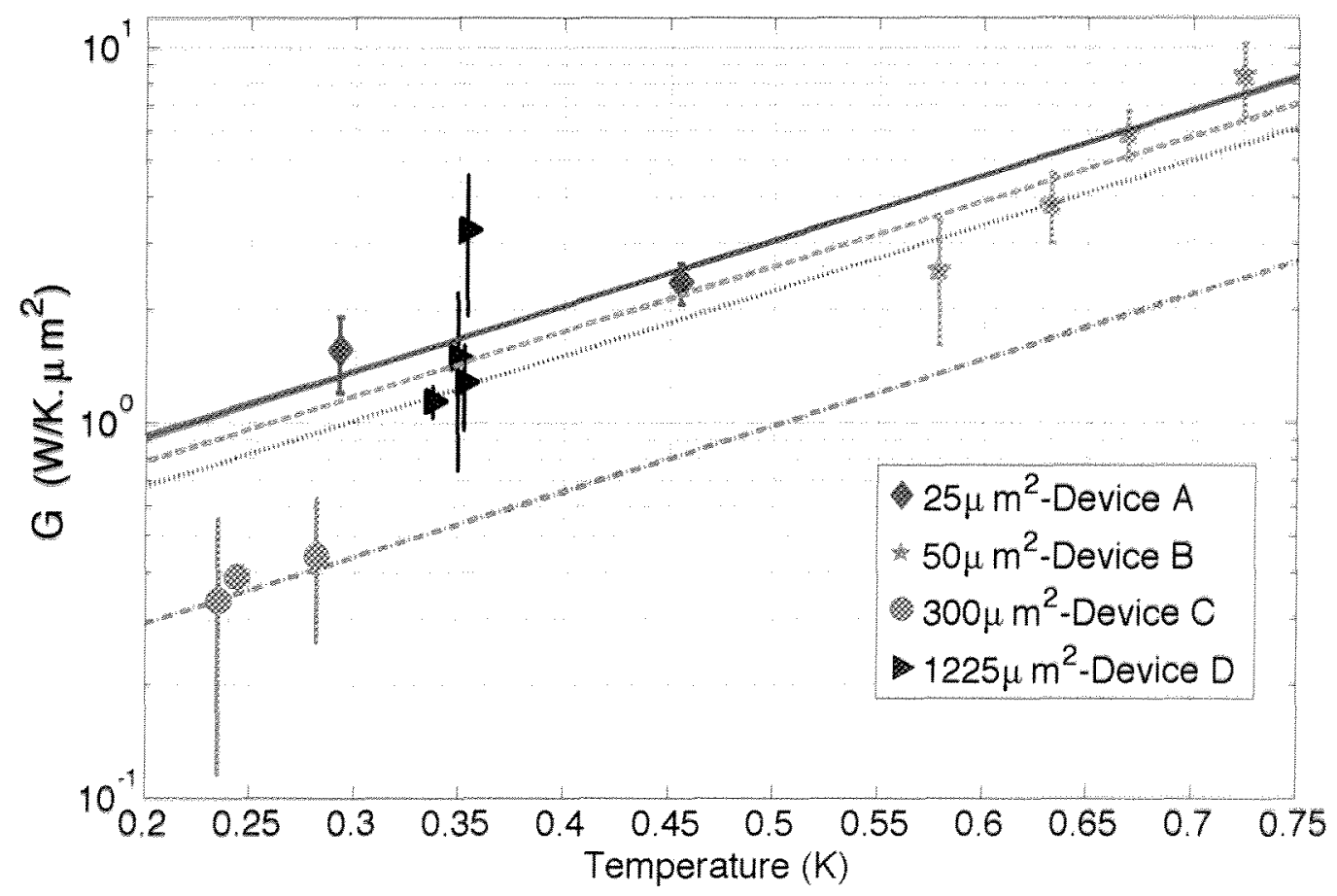

Figure 5. Thermal conductance of all devices and $T^{4}$ (straight line) fit to the $\mathrm{G}$ values. In order to decouple TES area from the $G$ values, in this plot, $G$ is divided by device area. $G$ values are plotted with error bars . 
The Noise Equivalent Power (NEP) derived from phonon fluctuations is straightforward:

$$
N E P \equiv \sqrt{4 k_{b} T^{2} G}=\sqrt{20 k_{b} \Sigma V T^{6}} .
$$

Assuming Device A geometry, and corresponding $G$ value, Figure 6 presents the calculated NEP for the detector. At 60 $\mathrm{mK}$, the achieved NEP is $1.65 \times 10^{-18} \mathrm{~W} / \sqrt{ } \mathrm{Hz}$.

The time constant of an EPD detector is given by:

$$
\tau_{e p} \equiv \frac{C_{e}}{G_{e p}}=\frac{\gamma T_{e} V}{5 \Sigma V T_{e}^{4}}=\frac{\gamma}{5 \Sigma T_{e}^{3}}
$$

where $\gamma$ is the Sommerfeld constant for electronic specific heat. For $\mathrm{Au}^{9}, \gamma \sim 71 \mathrm{~J} / \mathrm{K}^{2} \mathrm{~m}^{3}$. We estimate the time constant, $\tau_{e p} \approx 5 \mu \mathrm{s}$, which is compatible with a typical SQUID amplifier or time division SQUID multiplexer. The effects of thermal conductance and electrothermal feedback are major determinants of the time constant, but the electronic heat capacity also plays a major role. Using absorber structures contacting the TES, this electronic heat capacity has been tuned to be about ten times larger (see Table 2) than that of the TES element, slowing the device down from its intrinsic time constant of $\sim 5 \mu$ s to be compatible with a multiplexed SQUID.

Table 2. Electronic heat capacity $C_{e}$ for Bi absorber and Au layer in TES. Bi has area of $211.5 \mu \mathrm{m}^{2}$ and thickness of $0.8 \mu \mathrm{m}$, while, $\mathrm{Au}$ on TES has area of $25 \mu \mathrm{m}^{2}$ and thickness of $0.2 \mathrm{~nm} . \gamma$ and $\Sigma$ are obtained from literature. Heat capacity contribution of Mo is negligible; hence we have assumed heat capacity of Au as TES heat capacity.

\begin{tabular}{|l|l|l|l|l|}
\hline Material & $\begin{array}{l}\mathbf{V} \\
\left(\mathbf{m}^{3}\right)\end{array}$ & $\begin{array}{l}\sum \\
\left(\mathbf{W} / \mathbf{m}^{3} \mathbf{K}^{4}\right)\end{array}$ & $\begin{array}{l}\boldsymbol{\gamma} \\
\left(\mathbf{J} / \mathbf{K}^{2} \mathbf{m}^{3}\right)\end{array}$ & $\begin{array}{l}C_{e} \\
(\mathbf{J} / \mathbf{K})\end{array}$ \\
\hline Bi (Absorber) & $1.68 \times 10^{-10}$ & $0.24 \times 10^{9}$ & 0.39 & $2.09 \times 10^{-17}$ \\
\hline Au (TES) & $5 \times 10^{-21}$ & $5 \times 10^{8}$ & 71 & $1.11 \times 10^{-19}$ \\
\hline
\end{tabular}

As an aside, we did not observe a correlation between superconducting transition temperature, $T_{c}$, and the lead-to-lead distance in our measurements. The lead-to-lead distance for Device A and Device B is $4 \mu \mathrm{m}$, and for Device C and Device D is it $12 \mu \mathrm{m}$ and $36 \mu \mathrm{m}$, respectively.

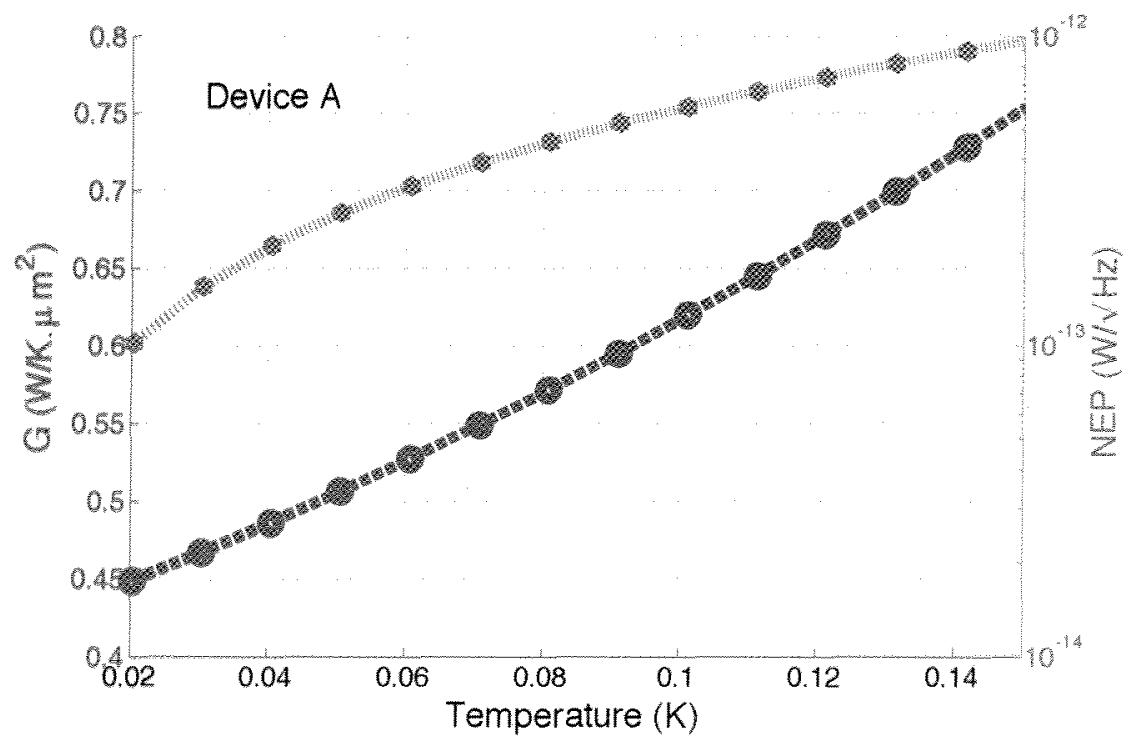

Figure 6. Measured G and calculated NEP for TES with Device A geometry. We aim to operate our detector at $100 \mathrm{mK}$ transition temperature. 


\section{CONCLUSIONS AND FUTURE WORK}

We have successfully fabricated TES with various geometries which exhibit thermal isolation that is dictated byelectronphonon coupling phenomena based upon the relationship between thermal conductance and temperature. The measured values of $\mathrm{G}$ are consistent with our initial calculations and published values ${ }^{8,9}$. With a $25 \mu \mathrm{m}^{2}$ sensor area, we estimate NEP to be $\sim 1.65 \times 10^{-18} \mathrm{~W} / \sqrt{ } \mathrm{Hz}$ and time constant of $\sim 5 \mu \mathrm{s}$.

We plan on fabricating devices with lower superconducting transition temperature and simultaneously testing different TES geometries e.g., the zebra normal metal stripes, superconducting imbedded structures in TES devices. The improvement in the TES designs and geometry will increase the sensitivity of detector by $30 \%$ to $50 \%$, and hence, estimated NEP is $\sim 2.5 \times 10^{-19} \mathrm{~W} / \mathrm{JHz}$, which is in the ZeptoWatts $/ \sqrt{ } \mathrm{Hz}$ sensitivity range. The antennas have been designed, simulated and produced for frequencies of $300 \mathrm{GHz}, 1 \mathrm{THz}$, and $3 \mathrm{THz}$. We plan on testing the antenna with optical input power and integrate them onto TES device. Recently, we have fabricated device with copper $(\mathrm{Cu})$ ground plane, and testing is planned in near future. We are also in the middle of installing SQUID setup in our cryogenic systems; hence we should be able to reduce the uncertainties of $G$ values. We are in the process of characterizing superconducting proximity effect for $\mathrm{Mo} / \mathrm{Au}$ films, and optismistic about achieveing required transition temperature. Such devices operating at lower temperature, e.g. $60 \mathrm{mK}$ will be ideal for low background measurements of millimeter and submillimeter wave astrophysics.

\section{REFERENCES}

[1] Irwin, K.D., "An application of electrothermal feedback for high resolution cryogenic particle detection", Applied Physics Letters 66 (15), pp. 1998-2000 (1995).

[2] Lee, A.T., Richards, P.L., Nam, S.W., Cabrera, B \& Irwin, K.D., "A superconducting bolometer with strong electrothermal feedback", APL, 69, pp.1801-1803 (1996).

[3] Benford, D.J., Ames, T.A., Chervenak, J.A., Grossman, E.N., Irwin, K.D., Khan, S.A., Maffei, B., Moseley, S.H., Pajot, F., Phillips, T.G., Renault, J.-C., Reintsema, C.D., Rioux, C., Shafer, R.A., Staguhn, J.G., Vastel, C. \& Voellmer, G.M., "First Astronomical Use of Multiplexed Transition Edge Bolometers", AIPC, 605, 589 (2002).

[4] Irwin, K.D., Hilton, G.C., Wollman, D.A. \& Martinis, J.M. "Thermal-response time of superconducting transitionedge microcalorimeters", JAP, 83 (8), p.3978-3985 (1998).

[5] Cabrera, B., R. M. Clarke, P. Colling, A. J. Miller, S. Nam, and R. W. Romani, "Detection of single infrared, optical, and ultraviolet photons using superconducting transition edge sensors", Appl. Phys. Lett., vol. 73, pp. 735737 (1998).

[6] Karasik, B.S., W. R. McGrath, M. E. Gershenson, and A. V. Sergeev, "Photon-noise-limited direct detector based on disorder-controlled electron heating", Journal of Appl. Physics, vol. 87, no. 10, pp. 7586-7588 (2000).

[7] Wei, J., Olaya, D., Karasik, B.S., Pereverzev, S.V., Sergeev, A.V. \& Gershenson, M.E., "Ultra-Sensitive HotElectron Nanobolometers for Terahertz Astrophysics", Nature Nanotechnology 3, 496 - 500 (2008).

[8] Ali, S., Timbie, P.T., Siddharth, M., McCammon, D., Nelms, K.L., Pathak, R., van der Weide, D.W., Allen, C.A., Abrahams, J. \& Chervenak, J.A., “Antenna-Coupled Transition-Edge Hot-Electron Microbolometers", Proc. SPIE, \#5498, 381 (2004).

[9] Barrentine, E. M., Timbie, P. T., Stevenson, T. R., Ali, S., Chervenak, J. A., Wollack, E., Moseley, S. H., Allen, C. A., Hseih, W. T., Miller, T. M., Benford, D. J., \&Brown, A. D., "Sensitivity Measurements of a Transition-Edge Hot-Electron Microbolometer for Millimeter-Wave Astrophysical Observations", J. Low Temp. Physics, 151, pp. 173-179 (2008).

[10] Brown, Ari-David; Benford, Dominic J.; Chervenak, James A.; Henry, Ross; Kletetschka, Gunther; Mikula, Vilem; Stevenson, Thomas R.; U.-Yen, Kongpop; Wollack, Edward J. The $13^{\text {th }}$ International Workshop on Low Temperature-LTD13. AIP Conference Proceedings, Volume 1185, pp. 326-329 (2009).

[11] M. Yu. Reizer and A. V. Sergeev, Zh. Eksp., Sov. Phys. JETP 63, 616 (1986); Teor. Fiz. 90, 1056 (1986). 